\title{
Intolerance of uncertainty, future time perspective, and self-control
}

\author{
Qing Yang ${ }^{\mathrm{a}, *}$, Kees van den Bos ${ }^{\mathrm{b}}$, Yaqin $\mathrm{Li}^{\mathrm{a}}$ \\ ${ }^{a}$ School of Psychology, Qufu Normal University, China \\ ${ }^{\mathrm{b}}$ Department of Psychology and School of Law, Utrecht University, Netherlands
}

\section{A R T I C L E I N F O}

\section{Keywords:}

Intolerance of uncertainty

Future time perspective

Self-control

Anxiety

Construal level theory

\begin{abstract}
A B S T R A C T
Intolerance of uncertainty (IU) is a transdiagnostic vulnerability factor for emotional disorders. Inspired by Construal Level Theory, the present research investigated the possibility that future time perspective (FTP) might be linked to lower levels of IU. After all, future-oriented construals can lead people to place more weight on longterm goals, leading to a better tolerance of current uncertainties. Results indeed revealed that higher FTP was associated with lower IU. Moreover, long-term goals generally involve more self-control than immediate gratification. In accordance with this line of reasoning, we explored whether self-control could both mediate and moderate the relationship between FTP and IU. Mediation analysis confirmed that higher FTP was associated with greater self-control, which in turn was linked to less IU. However, the moderation effect was not observed. The discussion explains how the current findings expand our knowledge of the nature of IU, suggesting theoretical and clinical implications for dealing with it.
\end{abstract}

\section{Introduction}

Real life is accompanied by plenty of ambiguity, and accepting some level of uncertainty is required to maintain psychological functioning. Intolerance of Uncertainty (IU) is a tendency to hold negative beliefs about uncertainty such that higher IU individuals perceive uncertain situations to be threatening and believe that uncertainty should be avoided. People with higher levels of IU find uncertainty to be stressful and fearful, and have difficulty enduring aversive experiences related to uncertainty (Buhr \& Dugas, 2002).

A concept that often is being treated as a synonym of IU is Intolerance of Ambiguity (IA), which also refers to the tendency that individuals perceive equivocal situation as a threat, resulting in some irrational thinking patterns and emotional responses (Houran \& Williams, 1998). Despite these commonalities, some researchers noted that IU and IA may be related to different time orientations such that "IU refers to the interpretation of a future event and IA to the interpretation of an ambiguous stimulus in the "here and now"' (Grenier et al., 2005, p. 596). This may explain why IU is relatively more involved in anxiety disorders because these disorders usually involve anticipation toward future bad outcomes (Grenier et al., 2005).

Studying IU is important, in part because IU can be linked to dysfunctional emotion regulation. Indeed, high levels of IU have been identified as transdiagnostic risk factors (i.e., a prominent feature across different disorders but not specific to any single disorder) for anxiety and depression symptoms, including generalized anxiety and obsessive compulsive and major depressive disorders (Carleton, 2016). Furthermore, IU is related to cognitive "myopia," as indicated by impulsive decision-making known as delay discounting. For example, higher IU individuals tend to choose smaller immediate rewards (even with lower probability) over larger delayed ones, because waiting in an uncertain state can be torturing for them (Luhmann et al., 2011).

In short, both the emotional and cognitive features of high levels of IU can harm psychological well-being and impede the pursuit of longterm important goals. It thus seems important to study what variables dampen higher IU to occur.

Inspired by Construal Level Theory (CLT; Trope \& Liberman, 2003), we propose that Future Time Perspective (FTP) may be a protective variable helping deal with IU. FTP refers to a general future-orientated time frame regarding to the planning for and achievement of future goals (Zimbardo \& Boyd, 1999). Individuals with higher FTP tend to focus on distant future outcomes rather than the present or near future. According to CLT, psychologically distant future objects tend to be represented on more abstract, higher-level construals than those are close in time (Trope \& Liberman, 2003). Subjectively distancing from targets such as uncertain events, or priming of abstract thoughts promotes individuals to think about the "broader picture", and emphasize the superordinate, immutable features of an event. Indeed, research

\footnotetext{
* Corresponding author at: School of Psychology, Qufu Normal University, No.57, JingXuan West Road, Qufu, Shandong 273165, China.

E-mail address: yq99550@gmail.com (Q. Yang).
} 
revealed that psychologically distancing or abstracting construals can reduce the affective intensity and valence of negative events, suggesting that FTP may help with effective emotion regulation (Williams et al., 2014). Related to this, FTP is associated with less anxiety and depression (Papastamatelou et al., 2015). Furthermore, by placing more weight on long-term goals, FTP may help for efficient goal pursuit (Fujita et al., 2006). For example, FTP is related to stronger preferences for larger, delayed gains (Daugherty \& Brase, 2010). This suggests that FTP may help to deal with emotional or cognitive dysfunctioning regarding high IU. We thus predict that higher FTP is associated with lower IU.

Importantly, the connection between FTP and IU may be influenced by other variables, especially by those that are closely related to emotion regulation and goal pursuit. One important variable is self-control, a component of individuals' ability to restrain undesirable thoughts or impulses, alter moods or emotions, to achieve optimal outcomes (Tangney et al., 2004). Higher self-control individuals may generally better deal with uncertainty-related emotional issues. For example, they may be better in tolerating uncertainty and successfully suppressing affective responses such as worry and anxiety (Tangney et al., 2004). Furthermore, higher self-control links to cognitive responses such as the inhibition of impulsive choices and to forego immediate, less-rewarded temptations (Fujita et al., 2006).

A concept relevant to self-control is Locus of Control (LoC), which refers to how people interpret the result of some behavior or event (Rotter, 1966). Specifically, internal control individuals tend to emphasize the importance of one's ability or efforts on the result of an event, while external control individuals attribute the result to luck or chance. Both self-control and LoC can promote individuals to take actions in achieving the desired goals such as life satisfaction and mental health, but they have difference in some way. For instance, self-control is a psychological process or ability to overcome unhealthy impulses, but LoC emphasizes the belief on the happening of things.

Self-control is also closely associated with FTP, because both selfcontrol and FTP are essentially future-oriented for goal achievement. After all, individuals with higher FTP tend to maintain temporal distance from or abstract with a situation, which can help individuals to better control themselves (Fujita et al., 2006) and make adjustments to achieve desirable results such as management of uncertainty (Price et al., 2017). Therefore, higher FTP may be associated with greater self-control, which in turn links to lower IU. That is, self-control may act as a mediator between FTP and IU.

Since higher self-control individuals may be more likely to foreseen a future picture and have stronger capacity to adopt FTP to facilitate goalsettings, it is possible that self-control may also serve as a moderator between FTP and IU such that the dampening effect of FTP on IU is stronger among high self-control individuals.

The current paper investigates whether self-control indeed can serve as a mediator, a moderator, or both as mediator and moderator between FTP and IU. Studying these potential mechanisms regarding the plausible roles of self-control can contribute to a novel understanding of the relation between FTP and IU.

\section{Method}

\subsection{Participants and procedure}

To detect even a small effect ( $r=0.20$ ) of FTP on IU with sufficient statistical power of 0.80 ( $\alpha=0.05$, two-tailed), we needed at least 194 participants. We enlarged this number and intended to recruit as many participants we could for detecting potential mediation or moderation effect. Finally, 677 Chinese college students (514 women and 163 men, mean age $=20.12$ years, $S D=1.49$, range $18-27$ ) voluntarily completed a paper-pencil test in exchange of small monetary compensation. This research was approved by the Human Research Ethics Committee of the first author's institution, and was not a part of broader empirical project that went beyond this paper. After reading and signing the consent form, participants completed demographic questions (e.g., age, gender), the main measures for assessing the three variables, and one additional question about their attitude toward money (i.e., for validating material that was intended to be used in future studies), which was not relevant to the present investigation. Then participants were thanked and debriefed.

\subsection{Measures}

\subsubsection{Future time perspective}

Based on the Zimbardo Time Perspective Inventory (Zimbardo \& Boyd, 1999) and related scales (e.g., Consideration of Future Consequence; Strathman et al., 1994), Song (2004) composed the Chinese version of General Future Time Perspective Scale. Zimbardo's 13-items subscale reveals a unidimensional structure of future orientation (e.g., "I complete projects on time by making steady progress"), while Song's 20items scale includes five factors: behavioral commitment (e.g., "I have goals everyday"), far-reach goal orientation (e.g., I often remind myself not to forget the most important goal of the future), future efficacy (e.g., "I believe I have the ability to build my own beautiful tomorrow"), future purpose consciousness (e.g., "I often feel that life has no purpose", reverse coded), and future image (e.g., "I know I have a lot of work ahead of me"), running from 1 "not at all characteristic of me" to 4 "entirely characteristic of me".

Although this Chinese scale has been used in previous research (e.g., Cheng et al., 2016), we do not know whether this scale was subjected to a back-translation procedure. Perhaps even more important, the structure validity of this tool was not tested and confirmed in the earlier studies. One solution to these problems, which we borrowed from same kind studies (e.g., Hong et al., 2019), was to check the items of the translated scales and see how we can improve the structure validity of the results reported in our study. Therefore, we ran item and factor analyses using the current sample to ensure good psychometric properties. Item analyses revealed that four items should be removed because they had vague description in Chinese (e.g., “我相当关注别人对我今后 发展的否定性评价”) and had low correlations with the scale's total score ( $r s=0.21-0.33$ ).

Furthermore, exploratory factor analyses (EFA; using principal component analysis extraction with varimax rotation) for the rest items revealed a three-factor structure that explained $52.11 \%$ of the total variance. Two items were dropped because they had high loadings on multiple factors. All of the remaining 14 items loaded above 0.55 on their corresponding factor.

Taken together, our analyses suggest that the original two factors of behavioral commitment and far-reach goal orientation in Song's scale are similar in meaning and can be composed as one factor (7 items). The original two factors of future efficacy and future image can also be composed as another factor ( 5 items). The original future purpose consciousness factor did not need to be altered (2 items).

Moreover, confirmatory factor analyses (CFA) revealed that the three-factor model could well fit the data: $\chi^{2} / d f=4.18$, Comparatively Fit Index $(\mathrm{CFI})=0.91$, Incremental Fit Index $(\mathrm{IFI})=0.92$, Bentler-Bonett Normed Fit Index $(\mathrm{NFI})=0.89$, Root Mean-Square Error of Approximation $($ RMSEA) $=0.07$, Standardized Root Mean Square Residual $(S R M R)=0.05$. When we ran CFA on the original 20 items, the fivefactor model (Song, 2004) revealed less satisfactory fit to the data, $\chi^{2}$ / $d f=4.49, \mathrm{CFI}=0.86, \mathrm{IFI}=0.86, \mathrm{NFI}=0.83, \mathrm{RMSEA}=0.07, \mathrm{SRMR}=$ 0.07 . Therefore, the three new dimensions with 14 items (Cronbach's $\alpha$ $=0.84$ ) were then used as indicators of FTP as a latent variable in subsequent analyses.

\subsubsection{Intolerance of uncertainty}

IU was measured by the widely used Intolerance of Uncertainty Scale (Buhr \& Dugas, 2002). The Chinese version was constructed by Dai et al. (2013), with a back-translation procedure. Buhr's original scale includes 27 items and four factors, while Dai's 21-items revised scale has two 
factors: uncertainty-related "negative belief and emotional experience" (e.g., "Uncertainty makes me uneasy, anxious, or stressed") and "behavioral dysfunction and negative self-reference" (e.g., "I can't stand being undecided about my future"), running from 1 "not at all characteristic of $m e$ " to 5 "entirely characteristic of $m e$ ". Previous research has used the Chinese scale (e.g., Yang et al., 2019), but did not test the structural validity. Therefore, to ensure its psychometric properties, we first ran item analyses and results showed that all items had high correlations with scale total score $(r s>0.50)$. Then EFA revealed a fourfactors structure that explained $54.71 \%$ of the total variance. Five items were dropped because they highly cross-loaded on different factors. The remaining items loaded above 0.50 on their corresponding factor. Lastly, CFA revealed that the four-factors model fitted the data very well: $\chi^{2} / d f=2.86, \mathrm{CFI}=0.95, \mathrm{IFI}=0.95, \mathrm{NFI}=0.93$, RMSEA $=$ 0.05 , SRMR $=0.04$, which seems fitting better than the two-factors solution (Dai et al., 2013), $\chi^{2} / d f=4.36$, CFI $=0.88$, IFI $=0.88$, NFI $=0.85$, RMSEA $=0.07$, SRMR $=0.05$. Generally, our revised scale was comparable to Buhr's four-factors solution, but was more concise. We then adopted the four new dimensions (16 items; Cronbach's $\alpha=0.89$ ) to represent IU as a latent variable.

\subsubsection{Self-control}

The Chinese version of Tangney' Self-Control Scale was used to measure self-control (Tangney et al., 2004; revised by Tan \& Guo, 2008). Tangney's scale includes 36 items and four factors (the fifth factor measures reliability), while Tan's 19-items has five factors, measuring impulse control (e.g., "I lose my temper too easily"), keeping healthy habits (e.g., "I have a hard time breaking bad habits"), inhibiting temptation (e.g., "I am good at resisting temptation"), focusing on work (e.g., "I have trouble concentrating"), and controlling entertainment (e. g., "I spend too much money"), running from 1 "not at all characteristic of $m e$ " to 5 "entirely characteristic of $m e$ ". The developers of the Chinese scale did not mention whether this scale was subjected to a backtranslation procedure, and the structural validity had not been confirmed in other studies. Again, to validate the revised scale, we checked the items and two were removed because of their low correlations with scale's total score ( $r$ s were around 0.30 ). Then, EFA revealed a three-factors structure that explained $45.58 \%$ of the total variance. We dropped two items because the high cross-loading problem on distinct factors. The rest 15 items loaded above 0.45 on their corresponding factor. CFA revealed that the three-factors solution provided overall better fit $\left(\chi^{2} / d f=2.18, \mathrm{CFI}=0.95, \mathrm{IFI}=0.95, \mathrm{NFI}=0.92\right.$, RMSEA $=$ 0.04 , SRMR $=0.04)$ than the five-factors solution $\left(\chi^{2} / d f=4.03, \mathrm{CFI}=\right.$ 0.85 , IFI $=0.85$, NFI $=0.82$, RMSEA $=0.07$, SRMR $=0.07$ ). The main adjustments were that the original two factors (focusing on work \& controlling entertainment) were similar in content and thus can be composed to one factor (4 items). The other two factors of keeping healthy habits and inhibiting temptation can also be combined to represent one factor (5 items). The impulse control factor was suggested to be remained (6 items). Ultimately, the three new dimensions (15 items; Cronbach's $\alpha=$ 0.82 ) were used to represent self-control as a latent variable.

\section{Results}

Consistent with our predictions, FTP was significantly and negatively associated with IU and positively associated with self-control. IU and

Table 1

Means, standard deviations (SD), and correlations between variables.

\begin{tabular}{lllll}
\hline Variables & Mean $(S D)$ & 1 & 2 & 3 \\
\hline 1. Future time perspective & $38.41(6.19)$ & - & & \\
2. Intolerance of uncertainty & $40.41(9.49)$ & $-0.21^{* * *}$ & - & \\
3. Self-control & $46.53(8.01)$ & $0.33^{* * *}$ & $-0.39^{* * *}$ & - \\
\hline
\end{tabular}

${ }_{* * * *}^{*} p<0.001$. These multiple correlations survived after Bonferroni correction with the threshold $p$ value lower than 0.016 . self-control were negatively correlated. See Table 1.

To get more insight into our results, we ran a built-in replication in the main analyses. That is, we would randomly split the whole sample into two pieces and used the first half for our first analyses $(N=338$ in Sample 1 with 250 females), and the other half for immediate replication ( $N=339$ in Sample 2 with 264 females). The two split samples were roughly comparable in demographic characteristics.

\subsection{Testing the hypothesized mediation model}

Structural equation modeling (SEM; performed by Amos 23.0) with maximum likelihood estimation and bias-corrected percentile bootstrap method (5000 iterations) were used to test the predicted mediation effect. Model fits were generally acceptable in Samples 1 and 2 (in parentheses, similarly hereinafter): $\chi^{2} / d f=4.53$ (5.09), CFI $=0.90(0.90)$, $\mathrm{IFI}=0.90(0.90), \mathrm{NFI}=0.86(0.84), \mathrm{RMSEA}=0.10(0.10), \mathrm{SRMR}=$ 0.08 (0.08).

Results in Table 2 and Fig. 1 show that higher FTP was linked to greater self-control, which predicted lower IU. However, the direct effect of FTP on IU was not significant. Importantly, the indirect effect through self-control was significant, suggesting that self-control fully mediated FTP's effect on IU. These findings were consistent across the two split samples. In addition, the ratio of the indirect effect to the total effect was around 50\% (44.36\% in Sample 1, and 52.02\% in Sample 2).

\subsection{Testing the hypothesized moderation model}

Next, SEM was used to conduct moderation analysis in the two samples, $\chi^{2} / d f=3.02(3.50)$, CFI $=0.90(0.90), \mathrm{IFI}=0.90(0.90)$, NFI $=0.85(0.82)$, RMSEA $=0.08(0.09)$, SRMR $=0.08(0.08)$. Results in Table 3 revealed that higher FTP was significantly associated with lower IU in Sample 1 (but not in Sample 2), higher self-control was significantly related to lower IU in both samples. However, the predicted interaction effect of FTP $\times$ self-control on IU was not significant in either sample.

\subsection{Testing alternative mediation models}

Since our data are cross-sectional, one may ask if there are other arrangements of the variables that also fit the data. One interesting and related question is whether FTP and self-control can switch their positions when influencing IU (Alternative mediation model A: Self-control $\rightarrow$ FTP $\rightarrow$ IU). This might be the case because higher self-control individuals tend to focus on long-term important goals (Tangney et al., 2004), thus implementing future temporal focus to facilitate this process (Fujita et al., 2006). The distancing and abstracting mindset then helps with effective emotion regulation such as better tolerating uncertainty (Williams et al., 2014). We tested this possibility with SEM analysis in the two samples: $\chi^{2} / d f=4.53(5.09), \mathrm{CFI}=0.90(0.90), \mathrm{IFI}=$ $0.90(0.90), \mathrm{NFI}=0.86(0.84)$, RMSEA $=0.10(0.10), \mathrm{SRMR}=0.08$ (0.08). Results showed that the mediating effect of FTP in the relationship between self-control and IU was significant in Sample 1 (indirect effect $=-0.07, p=0.022, \mathrm{CI}=-0.19$ to -0.01 ), but this finding was not replicated in Sample 2 (indirect effect $=-0.10, p=0.222, \mathrm{CI}=-0.30$ to 0.11 ).

Another interesting question is whether IU can predict FTP through the effect of self-control (Alternative mediation model B: IU $\rightarrow$ Self-control $\rightarrow$ FTP). This can be possible because higher IU individuals may be those who have less feeling of control over their life (Carleton, 2016), which in turn tempt them into grasping what is right in front of them (Luhmann et al., 2011), leading to impaired FTP. Again, we ran SEM analysis in the two samples, $\chi^{2} / d f=4.53$ (5.09), CFI $=0.90(0.90)$, IFI $=$ $0.90(0.90), \mathrm{NFI}=0.86(0.84)$, RMSEA $=0.10(0.10), \mathrm{SRMR}=0.08$ (0.08). We did find support for this assumption in one sample (Sample 2: indirect effect $=-0.09, p=0.024, \mathrm{CI}=-0.16$ to -0.02 ), but not in another sample (Sample 1: indirect effect $=-0.04, p=0.533, \mathrm{CI}=$ 
Table 2

Summary of the model paths from the hypothesized mediation analysis.

\begin{tabular}{|c|c|c|c|c|c|c|}
\hline \multirow[t]{2}{*}{ Model paths } & & \multirow[t]{2}{*}{ Standardized estimate $(\beta)$} & \multirow[t]{2}{*}{$p$} & \multicolumn{2}{|l|}{$95 \% \mathrm{CI}$} & \multirow[t]{2}{*}{$R^{2}{ }_{\text {med }}$} \\
\hline & & & & Lower & Upper & \\
\hline \multirow[t]{2}{*}{ FTP $\rightarrow$ Self-control } & Sample1 & 0.27 & 0.011 & 0.03 & 0.49 & - \\
\hline & Sample2 & 0.46 & 0.009 & 0.14 & 0.65 & - \\
\hline \multirow{2}{*}{ Self-control $\rightarrow$ IU } & Sample1 & -0.54 & 0.001 & -0.67 & -0.39 & - \\
\hline & Sample2 & -0.35 & 0.009 & -0.52 & -0.13 & - \\
\hline \multirow[t]{2}{*}{$\mathrm{FTP} \rightarrow \mathrm{IU}$} & Sample1 & -0.19 & 0.061 & -0.31 & 0.02 & - \\
\hline & Sample2 & -0.15 & 0.368 & -0.33 & 0.23 & - \\
\hline \multirow[t]{2}{*}{ FTP $\rightarrow$ Self-control $\rightarrow$ IU } & Sample1 & -0.23 & 0.010 & -0.54 & -0.02 & 0.04 \\
\hline & Sample2 & -0.30 & 0.008 & -0.67 & -0.08 & 0.04 \\
\hline
\end{tabular}

Note:95\% CI $=95 \%$ confidence interval. $R^{2}{ }_{\text {med }}$ represents the effect size of mediation path.

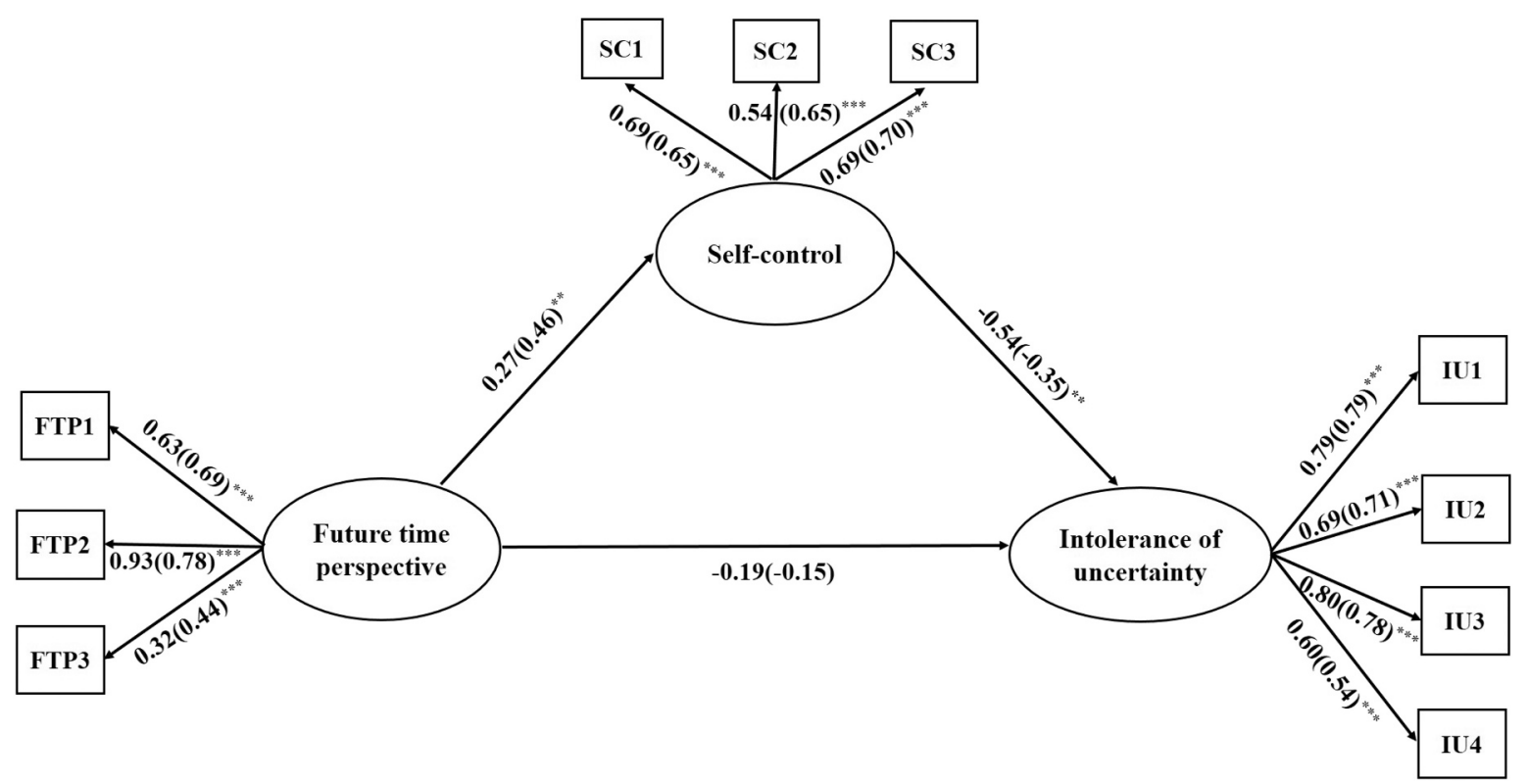

Fig. 1. Self-control mediates the effect of FTP on IU. Standardized path coefficients in Sample $1 \& 2$ (in parentheses) are shown. **p $<0.01$, ***p<0.001.

Table 3

Summary of the model paths from the hypothesized moderation analysis.

\begin{tabular}{|c|c|c|c|c|c|c|}
\hline \multirow[t]{2}{*}{ Model paths } & & \multirow[t]{2}{*}{ Unstandardized estimate (b) } & \multirow[t]{2}{*}{$p$} & \multicolumn{2}{|l|}{$95 \% \mathrm{CI}$} & \multirow[t]{2}{*}{$s r^{2}$} \\
\hline & & & & Lower & Upper & \\
\hline \multirow[t]{2}{*}{ FTP $\rightarrow$ IU } & Sample1 & -0.34 & 0.039 & -0.65 & -0.03 & - \\
\hline & Sample2 & -0.26 & 0.355 & -0.73 & 0.26 & - \\
\hline \multirow[t]{2}{*}{ Self-control $\rightarrow$ IU } & Sample1 & -1.07 & 0.002 & -1.53 & -0.66 & - \\
\hline & Sample2 & -0.72 & 0.021 & -1.20 & -0.21 & - \\
\hline \multirow[t]{2}{*}{ FTP $\times$ Self-control $\rightarrow$ IU } & Sample1 & -0.32 & 0.193 & -5.83 & 0.19 & $0.53 \%$ \\
\hline & Sample2 & -0.32 & 0.103 & -1.31 & 0.12 & $0.35 \%$ \\
\hline
\end{tabular}

Note: $s r^{2}$ represents the effect size of moderation path.

\section{-0.16 to 0.09$)$.}

Overall, the two alternative mediation models seemed not replicable and thus less supported by our current data set. In contrast, our main hypothesized mediation model was consistently and strongly supported.

\section{Discussion}

The present study may be one of the first to investigate both the potential relationship between FTP and IU and the mediation and moderation mechanisms through self-control. Our results revealed that higher FTP is significantly related with lower IU. This is consistent with previous studies that reported FTP was associated with less emotional dysregulation symptoms such as anxiety and depression
(Papastamatelou et al., 2015) and less impulsivity on choosing immediate, less-rewarded options (Daugherty \& Brase, 2010).

Furthermore, our mediation hypothesis was supported, revealing that self-control can fully mediate the relation between FTP and IU. Our support for an association between FTP and self-control is in line with the idea that a future-directed construal enhances people's appreciation for the broader, primary features of an event, which in turn promotes self-control (Fujita et al., 2006). Further, individuals with high selfcontrol are able to regulate their emotions (e.g., worry, anxiety) and associated behaviors (e.g., impulsive decision-making) under conditions of uncertainty. The full mediation effect pattern suggests that FTP and self-control may not work in isolation in influencing IU, but may be inherently related to each other as suggested by CLT (Trope \& Liberman, 
2003). We also compared the hypothesized model to two alternative mediation models, and the results revealed stronger support for the hypothesized one, suggesting that IU may be better predicted by FTP through the role of self-control, not the other way around.

We found little support for the moderation hypothesis. Although we proposed that people with higher self-control may be more likely to focus on future important goals (Tangney et al., 2004), and to adopt a future orientation to deal with the current uncertainties at hand, the built-in replication method did not confirm this. A straightforward explanation is that the split samples were not large enough to allow for robustly observing the moderation effect (e.g., participants that scored beyond $M \pm 1 S D$ were relatively few). We look forward future studies with larger samples to testing the possibilities. Another explanation is that self-control may be more suitable as a mediator (rather than a moderator), because self-control is correlated with and can be affected by FTP, as suggested by CLT and previous research (e.g., Fujita et al., 2006).

The present research adopted CLT to interpret the relations of the variables. But it is still possible that some other theoretical frameworks may also explain our findings. For instance, the Theory of Planned Behavior (TOPB) proposed by Ajzen (1991) suggests that behavioral achievement is predicted by behavioral intention, together with perceived behavioral control. Specifically, the success of IU dampening may be more likely to happen if one has stronger intention to overcome uncertainty-related negative responses (i.e., with better plan to achieve that goal, represented by FTP), and has higher perceived control to realize this goal (i.e., represented by self-control). Despite this, TOPB deals less with temporal orientation, such that we do not exactly know how a behavior would be differently predicted by near vs. distant future mindsets. It is also unclear how one's perceived control over behavior would be affected by the intention toward that behavior (i.e., TOPB does not offer prediction on this). Instead, CLT provides clear prediction on how temporal distance affects individuals' responses to future events, and also suggests its effect on self-control through abstracting mindset. Notwithstanding, future research is needed to test the efficacy of different theoretical frameworks in explaining the relations among FTP, self-control and IU.

Taken together, FTP and self-control seem like two protective variables contributing to lower IU. Interventions that focus on practices of future orientation and self-control may effectively regulate cognitive and emotional dysfunctional reactions to uncertainty. First, a futureoriented mindset may help for the cognitive reappraisal of uncertain events, for example, promoting people to think about the "broader picture" and positive side of the event, rather than treating it as a threat. These processes eventually contribute to effective emotion regulation and inhibiting impulsive decision-making, having people better live with uncertainties. Second, the mediating effect of self-control implies that FTP alone may not be enough to deal with IU, because mere future thoughts can lead to wishful thinking or fantasy, which is detrimental to goal pursuit (Oettingen \& Mayer, 2002). Overall, having a stronger future orientation (e.g., set up a plan) in conjunction with a high level of self-control (e.g., execute the plan) may provide valuable resources to overcome dispositional cognitive processes that otherwise may lead to less tolerance of uncertainty. Third, since IU is a transdiagnostic vulnerability factor for anxiety and depressive disorders (Carleton, 2016), the present findings may have implications for clinical practice. Previous research has shown that individuals with emotional disorders tend to interpret ambiguity in consistent, negative ways (Hirsch et al., 2016), training of FTP (e.g., by practicing episodic simulation with concrete imagery and procedures toward a goal) seems like a simple way for reducing interpretation biases and intervention for emotional disorders. Moreover, practices of self-control skills (e.g., monitor, evaluate and reinforce one's behavior toward a goal) may facilitate FTP's positive effect on dealing with these symptoms.

Due to our cross-sectional design, we cannot draw conclusions about the causality of the relations among the variables, future research should use longitudinal or experimental design to investigate the long-term effects of trait time perspective and self-control on IU to establish the causal links. Despite the limitation, the present findings show a promising avenue for future studies that people may better live with uncertainties by adopting an adaptive future-oriented mindset.

\section{CRediT authorship contribution statement}

Qing Yang: Conceptualization, Investigation, Methodology, Formal analysis, Writing - original draft, Writing - review \& editing. Kees van den Bos: Writing - original draft, Writing - review \& editing. Yaqin Li: Writing - review \& editing.

\section{Declaration of competing interest}

None.

\section{Acknowledgements}

This work was supported by the Youth Project of the 13th Five-Year Plan of Education Science in Shandong Province, titled "The effects of college students' materialism on well-being and coping strategies" (Grant Number: 2020QZD003).

\section{References}

Ajzen, I. (1991). The theory of planned behavior. Organizational Behavior and Human Decision Processes, 50, 179-211.

Buhr, K., \& Dugas, M. J. (2002). The intolerance of uncertainty scale: Psychometric properties of the English version. Behaviour Research and Therapy, 40, 931-945.

Carleton, R. N. (2016). Into the unknown: A review and synthesis of contemporary models involving uncertainty. Journal of Anxiety Disorders, 39, 30-43.

Cheng, C., Yang, L., Chen, Y., Zou, H., Su, Y., \& Fan, X. (2016). Attributions, future time perspective and career maturity in nursing undergraduates: Correlational study design. BMC Medical Education, 16, 26-33.

Dai, B., Zhang, G., \& Liu, H. (2013). Reliability and validity of the Chinese revised version of the intolerance of uncertainty scale. Chinese Journal of Behavioral Medicine and Brain Science, 22, 949-951.

Daugherty, J. R., \& Brase, G. L. (2010). Taking time to be healthy: Predicting health behaviors with delay discounting and time perspective. Personality and Individual Differences, 48, 202-207.

Fujita, K., Trope, Y., Liberman, N., \& Levin-Sagi, M. (2006). Construal levels and selfcontrol. Journal of Personality and Social Psychology, 90, 351-367.

Grenier, S., Barrette, A. M., \& Ladouceur, R. (2005). Intolerance of uncertainty and intolerance of ambiguity: Similarities and differences. Personality and Individual Differences, 39, 593-600.

Hirsch, C. R., Meeten, F., Krahé, C., \& Reeder, C. (2016). Resolving ambiguity in emotional disorders: The nature and role of interpretation biases. Annual Review of Clinical Psychology, 12, 281-305.

Hong, W., Liu, R. D., Ding, Y., Oei, T. P., Zhen, R., \& Jiang, S. (2019). Parents' phubbing and problematic mobile phone use: The roles of the parent-child relationship and children's self-esteem. Cyberpsychology, Behavior and Social Networking, 22, 779-786.

Houran, J., \& Williams, C. (1998). Relation of tolerance of ambiguity to global and specific paranormal experience. Psychological Reports, 83, 807-818.

Luhmann, C. C., Ishida, K., \& Hajcak, G. (2011). Intolerance of uncertainty and decisions about delayed, probabilistic rewards. Behavior Therapy, 42, 378-386.

Oettingen, G., \& Mayer, D. (2002). The motivating function of thinking about the future: Expectations versus fantasies. Journal of Personality and Social Psychology, 83, $1198-1212$.

Papastamatelou, J., Unger, A., Giotakos, O., \& Athanasiadou, F. (2015). Is time perspective a predictor of anxiety and perceived stress? Some preliminary results from Greece. Psychological Studies, 60, 468-477.

Price, M., Higgs, S., \& Lee, M. (2017). Self-control mediates the relationship between time perspective and BMI. Appetite, 108, 156-160.

Rotter, J. B. (1966). Generalized expectancies for internal versus external control of reinforcement. Psychological Monographs: General and Applied, 80, 1-28.

Song, Q. (2004). Theoretical and empirical research on future time perspective of university students. Doctoral dissertation: Southwest University, China.

Strathman, A., Gleicher, F., Boninger, D. S., \& Edwards, C. S. (1994). The consideration of future consequences: Weighing immediate and distant outcomes of behavior. Journal of Personality and Social Psychology, 66, 742-752.

Tan, S. H., \& Guo, Y. Y. (2008). Revision of self-control scale for Chinese college students. Chinese Journal of Clinical Psychology, 16, 468-470.

Tangney, J. P., Baumeister, R. F., \& Boone, A. L. (2004). High self-control predicts good adjustment, less pathology, better grades, and interpersonal success. Journal of Personality, 72, 271-324.

Trope, Y., \& Liberman, N. (2003). Temporal construal. Psychological Review, 110, 403-421. 
Williams, L. E., Stein, R., \& Galguera, L. (2014). The distinct affective consequences of psychological distance and construal level. Journal of Consumer Research, 40, 1123-1138.

Yang, Q., Ybarra, O., Van den Bos, K., Zhao, Y., Guan, L., Cao, Y., ... Huang, X. (2019). Neurophysiological and behavioral evidence that self-uncertainty salience increases self-esteem striving. Biological Psychology, 143, 62-73.
Zimbardo, P. G., \& Boyd, J. N. (1999). Putting time in perspective: A valid, reliable individual-differences metric. Journal of Personality and Social Psychology, 77, 1271-1288. 